\title{
Large Impact of obesity on the disposition of ivermectin, moxidectin and eprinomectin in a canine model: relevance for COVID-19 patients.
}

\author{
Alain Bousquet-Melou ${ }^{1}$, Anne Lespine ${ }^{2}$, Jean-François Sutra ${ }^{2}$, Isabelle Bargues ${ }^{1}$, and \\ Pierre Louis Toutain ${ }^{1}$ \\ ${ }^{1}$ Ecole nationale veterinaire de Toulouse \\ ${ }^{2}$ INRAE
}

January 12, 2021

\begin{abstract}
Background and Purpose: Based on in vitro data, ivermectin (IVM) has been proposed for the prevention and treatment of COVID-19, a condition for which obesity is a major risk factor. IVM dosage is based on total body weight and there are no recommendations to adjust dosage in obese patients. The objective of this study was to establish, in a canine model, the influence of obesity on the clearance and steady-state volume of distribution of IVM and two analog compounds, moxidectin (MOX) and eprinomectin (EPR). Experimental Approach: An experimental model of obesity in dogs was based on a high calorie diet. IVM, MOX and EPR were administered intravenously, simultaneously in combination, to a single group of dogs in two circumstances, during a control period and when body weight had been increased by $50 \%$. Key Results: In obese dogs, clearance, expressed in absolute values (L/day), was not modified for MOX and reduced for IVM and EPR, compared to the initial control state. When scaled by body weight (L/day/kg), plasma clearance was reduced by 42,55 and $63 \%$, for MOX, IVM and EPR, respectively. In contrast, the steady-state volume of distribution was markedly increased in absolute values (L) by obesity. Conclusion and Implications: For IVM and MOX, the obese dog model suggests that the maintenance dose should not be adjusted by total body weight in the obese subject but should be based on lean body weight. On the other hand, the loading dose should be computed based on the total body weight of the obese subject.
\end{abstract}

\section{Hosted file}

Ivermectin for submission.pdf available at https://authorea.com/users/389061/articles/503709large-impact-of-obesity-on-the-disposition-of-ivermectin-moxidectin-and-eprinomectin-ina-canine-model-relevance-for-covid-19-patients 